\title{
STUDY OF PATHOGENIC FACTORS OF E.COLI ISOLATED FROM PATIENTS WITH PERITONITIS
}

DOI: 10.36740/WLek202001114

\author{
Olha Yu. Kosilova' ${ }^{1}$ Oleksandra 0. Vovk' ${ }^{1}$, Nataliia M. Katelevska' ${ }^{1}$, Tetiana P. Osolodchenko², \\ Svitlana V. Ponomarenko ${ }^{2}$, Viacheslav Yu.Vdovichenko ${ }^{1}$ \\ 'KHARKIV NATIONAL MEDICAL UNIVERSITY, KHARKIV, UKRAINE \\ 2STATE INSTITUTION «I. MECHNIKOV INSTITUTE OF MICROBIOLOGY AND IMMUNOLOGY NATIONAL ACADEMY OF MEDICAL SCIENCES OF UKRAINE», \\ KHARKIV, UKRAINE
}

\begin{abstract}
The aim: To study the biological properties of museum and clinical strains of E.coli isolated from patients with peritonitis. Materials and methods: It was used 94 strains (clinical, museum and reference). The ability of $E$. coli to adhere was investigated by hemadhezive method to formal human erythrocytes of 0 (I) Rh-positive blood group. The study measured the ability of microorganisms to produce gelatinaze, caseinase, fibrinolysin, hemolysin. To control of the enzyme activity the positive and negative control with reference strains were used. Synchronisation of cultures activity before experiments was achieved by one-time effect of low temperature $(+4 \mathrm{C})$ during 30 minutes. Results: To investigate the pathogenic factors of $E$. coli we carried out determining of proteolytic, gelatinous, caseinous, fibrinolytic, haemolytic and adhesion properties. Conclusions: In our investigation pathogen icspecies of Esherihies are virtually indistinguishable from representatives of normal microflora on its morphological, biochemical and cultural properties. During investigation of serological properties of selected of $E$. coli strains $(n=94) 65.8 \%$ of pathogenic serotypes were revealed. Moreover, all marked E. coli isolated from the abdominal cavity of children and adults, as well as museum strains related to enteropathogenic E. coli (0127: K63, 033: K-) and 1 - to enteroinvazive Escherichia coli (0144: K-).
\end{abstract}

KEY WORDS: E.coli, peritonitis, biological properties

Wiad Lek. 2020;73(1):78-82

\section{INTRODUCTION}

The human's colon maintains a microbial density approaching $10^{12}$ organisms per gram of feces, representing a perfectly balanced ecosystem. The commensal microbiota, derived from the Latin commensal and meant sharing a table, consists of more than 400 species and lives in perfect harmony with the human intestine $[1,2]$.

Escherichia coli is a commensal inhabitant of the intestinal tracts of healthy humans and many animal species, but it can also cause a wide range of diseases, ranging from diarrhea to extraintestinal infections $[2,3]$. But $E$. coli is more than just a laboratory workhorse or harmless intestinal inhabitant; it can also be a highly versatile, and frequently deadly, pathogen. Extraintestinal pathogenic Escherichia coli (ExPEC), the specialized strains of E. coli that cause most extraintestinal E. coli infections, represents a major but little-appreciated health threat. Although the reasons for their evolution remain mysterious, by virtue of their numerous virulence traits ExPEC clearly possess a unique ability to cause disease outside the host intestinal tract. Broader appreciation of the existence and importance of ExPEC and better understandings of their distinctive virulence mechanisms, reservoirs, and transmission pathways may lead to effective preventive interventions against the morbid and costly infections ExPEC cause $[4,5]$.
Escherichia coli is one of the most common isolates in clinical microbiology laboratories and classified into three major groups: commensal strains, intestinal pathogenic strains, and extraintestinal pathogenic strains, according to their biological significance to humans $[5,6,7]$. $\mathrm{Pu}-$ rulent peritonitis in modern conditions differs from the bacteriological point of view of a combined flora with ever-increasing pathogenic properties. At the same time, Escherichia coli retains the dominant role in the microbial etiology of peritonitis [8].

As pathogenic factors we define adaptive mechanisms of infectious diseases. First of all you should decide on such a fundamental property of bacteria as pathogenic.

The presence of microbial pathogenicity factors is important for assessing the etiological significance.

For E. coli, pathogenicity is not a species sign and is not related to a specific serogroup. They are able to realize their pathogenic potential and cause disorders in the human body, limited only by those genetic determinants that have a specific strain of $E$. coli.

\section{THE AIM}

To study the biological properties of museum and clinical strains of $E$. coli isolated from patients with peritonitis. 


\section{MATERIALS AND METHODS}

It was used 94 strains (clinical, museum's and reference). The ability of $E$. coli to adhesion was tested with hemadhezive method on formalinized human erythrocytes of 0 (I) Rh-positive blood group [9].

Within the research we measured the ability of microorganisms to produce gelatinase, caseinase, fibrinolysin, hemolysin.

Positive and negative control with reference strains were used to control of the enzyme activity.

Synchronisation of cultures activity before experiments was achieved with simultaneous time effect of low temperature $(+4 \mathrm{C})$ within 30 minutes.

\section{RESULTS AND DISCUSSION}

To investigate the pathogenic factors of $E$. coli we carried out proteolytic, gelatinous, caseinous, fibrinolytic activities, as well as adhesion properties.

It was found that all investigated strains of E. coli, 94 in number, had proteolytic activity. This high degree of proteolytic activity wasn't found. All clinical and museum strains were weak active.

In addition to discussed above biological properties of obtained bacteria, the fibrinolytic activity was determined. This factor of pathogenicity aimed at dissolving fibrin, that limits local inflammatory center, and thus contributes to the generalization of the pathological process.

In the study on fibrinolytic activity of all clinical strains and isolated from relatively healthy individuals had a negative result. This statement corresponds to the date given in scientific literature due to which the strains of $E$. coli do not have the capacity for fibrinolytic activity.

In the study of pathogenicity factors the great importance is given to hemolytic activity. It is connected with cytotoxicity, here with the lysis direction, which action is not limited only on erythrocytes, but it also spreads on other cell types. The ability of many strains of $E$. coli to cause haemolysis of red blood cells of humans and animals is connected with the presence of $\alpha$-hemolysin gene in cells that is a major factor of $E$. coli pathogenicity. Hemolytic active is shown up with thermally labile toxins, extracellular $\beta$-hemolysin and enterohemolizyn that localizes in the outer membrane of the bacterial cell and cytolizyn A. They "drill" plazmalema of red blood cells as well as other cells including phagocytes by making them viable.

It is known from the literature that $E$. coli strains with plasmid extraintestinal localization have $\alpha$ - hemolysin $[10,11,12]$. Produced cytotoxin strains are resistant to chemotherapeutic medicines and they are particularly dangerous [13].

We studied the hemolytic activity in E. coli strains extracted from children and adult patients with peritonitis, and also museum and strains that was isolated from relatively healthy people (group).

During analyzing the results of the determination of the hemolytic activity in deleted strains $E$. coli $(\mathrm{n}=94)$ it was found that $4(4,2 \pm 2,0) \%$ strains isolated from adults were able to produce $\alpha-$ hemolysin.
In all the clinical strains of $E$. coli being extracted from children and adults, as well as museum and isolated from relatively healthy people we found ability to gelatinase activity. It was established that in $4(16,0 \pm 7,3) \%$ of strains, that was removed from adults, gelatinase activity was sharply positive with a positive result - $10(40,0 \pm 9,7) \%$. Also weak active strains- $5(20,0 \pm 8,0) \%$ were found. We got a negative result in $6(24,0 \pm 8,5) \%$ strains .

In the study of the museum strains of $E$. coli it was found that $2(10,5 \pm 7,0) \%$ strains were with high positive activity of gelatinase $-13(68,4 \pm 10,6) \%$ strains. There were strains with weak activity of gelatinase $4(21,0 \pm 9,3) \%$ and those which had a negative result $-1(5,2 \pm 5,0) \%$ strain. It should be noted that strains of $E$. coli extracted from children didn't have little and sharply positive gelatinase activity, but there were $2(8,0 \pm 5,4) \%$ cases in which strains gave positive results in 48 hours, indicating a weak gelatinase activity. The vast number of strains were made up with $E$. coli, which had a positive activity gelatinase $22(88,0 \pm$ $6,4) \%$. Among the studied strains $1(4,0 \pm 3,9) \%$ was with negative result.

During analyzing gelatinase activity in strains extracted from relatively healthy people, positive strains were $2(8,0$ $\pm 5,4) \%$, and weakly positive $-1(4,0 \pm 3,9) \%$. However, the interesting thing was that negative strains and those that gave positive results in 48 hours turned out the same number of $10(40,0 \pm 9,7) \%$.

The value of adhesive characteristics of bacteria for a microorganism could be considered from two positions. On the one hand, the adhesive capacity of indigen microflora is one of the factors in the realization of resistance, the intestinal mucosa colonization and obstacles for attachment to receptors of the mucosal pathogens. On the other hand, the development of dysbiotic violations of adhesive properties of opportunistic microflora is considered as a pathogenicity factor because they allow microbes to get fixed on the surface of the skin or mucous membranes and colonize this biotype by reaching a certain level of population. Now it is shown the adhesive nature of the changes of microbes characteristics in development of microecological disorders of oropharynx and nasapharynx, vagina; proposed approaches to lowering of the adhesion of conditional - pathogenic bacteria were proposed. At the same time, data on the biological properties of intestinal microbiota representatives were quite a few that is probably due to species diversity of biotope.

From the literary sources it is known that adhesion plays an important role in the development of infection, it also determines its beginning and promotes the further invasion of microorganisms in the host tissue. Pathogenic potential of microorganisms, when translocated from the place of natural habitation to other biotopes significantly increases. We have studied the adhesive activity of strains of $E$. coli extracted from children and adult patients with peritonitis, and museum strains isolated from relatively healthy people. The results of the study of adhesive properties E. coli, extracted from patients with peritonitis, museum strains and strains isolated from relatively healthy people are shown in Table. I. 
Table I. Level adhesivity of strains of E.coli isolated from the patients with peritonitis, museum strains and strains isolated from relatively healthy people (group)

\begin{tabular}{|c|c|c|c|c|}
\hline Indices of adhesion & $\begin{array}{l}\text { E.coli strains isolated } \\
\text { from children } \\
(n=25), \\
M \pm m\end{array}$ & $\begin{array}{l}\text { E.coli strains isolated } \\
\text { from adults } \\
(n=25), \\
M \pm m\end{array}$ & $\begin{array}{c}\text { Museum strains } \\
\text { of E.coli } \\
(n=19), \\
M \pm m\end{array}$ & $\begin{array}{c}\text { E.coli strains are } \\
\text { isolated from } \\
\text { conventionally healthy } \\
\text { people (comparison } \\
\text { group) }(\mathrm{n}=\mathbf{2 5}), \mathrm{M} \pm \mathrm{m}\end{array}$ \\
\hline Average adhesion & $3,3 \pm 0,3$ & $3,2 \pm 0,3$ & $1,9 \pm 0,2$ & $2,5 \pm 0,2$ \\
\hline Adhesion coefficient (\%) (C) & $41,1 \pm 9,8$ & $35,4 \pm 9,6$ & $30,0 \pm 10,5$ & $28,3 \pm 9,0$ \\
\hline Adhesion index & $4,8 \pm 0,4^{*}$ & $4,6 \pm 0,3$ & $2,7 \pm 0,3$ & $3,3 \pm 0,3^{*}$ \\
\hline
\end{tabular}

Note: the difference is statistically significant parameters $(p<0.001)$

Table II. Characteristics of enzymatic activity of strains of $E$. coli, isolated from children and the adult patients with peritonitis, museums and strains isolated from healthy conventionally (comparison group) $(n=94)$

\begin{tabular}{cc}
\hline Test or substrate & Percentage of positive strains, $\%$ \\
\hline Citratus of Simons & 0 \\
\hline Glucose $(A, G)$ & 100 \\
\hline Hydrogen sulfide & 91,5 \\
\hline Indole & 90,4 \\
\hline Lactose $(A)$ & 98 \\
\hline Lysinedecarboxylase & 89,4 \\
\hline Mobility & 87,2 \\
\hline Ornithinedecarboxylase & 100 \\
\hline Reaction with methyl red & 89,4 \\
\hline Sorbitol (A) & 0 \\
\hline Urea & 0 \\
\hline
\end{tabular}

Note. $A G$ - acid and gas, $A$ - acid.

The study found that the average number of red blood cells, which were involved in the process of adhesion (C) of E. coli strains extracted from adult patients with peritonitis, museum strains and strains isolated from relatively healthy people (group) did not differ significantly ( $p>0.05$ ).

During analyzing the results of adhesive properties of $E$. coli strains isolated from adult patients with peritonitis the following results were obtained: 5 strains had high adhesive activity corresponding to $(20,0 \pm 4,4) \%$, average -20 $(80.0 \pm 8,0) \%$ of 25 studied strains. For comparison group strains (strains conventionally extracted from the intestine of healthy people), the average was adhesiveness in $2(8,0$ $\pm 2,8) \%$ of cases, the proportion of strains of low-adhesive E. coli of this group was $23(92,0 \pm 8,4) \%$.

In the study of strains of $E$. coli isolated from children suffering of peritonitis we can say that strains with secondary and high adhesiveness were turned out $12(48,0 \pm$ $6,5) \%$ and $13(52 \pm 6,7) \%$ in accordance.

Data analysis of the study of adhesiveness museum strains of $E$. coli, showed that 5 strains $(26,3 \pm 5,0) \%$ were with high adhesiveness and 15 of 19 strains $(78,9 \pm 8,2) \%$ were with middle adhesiveness.

Thus, all studied of strains of $E$. coli had adhesive properties. Higher rates of adhesion of E.coli strains isolated from children and adult patients with peritonitis, in comparison with strains isolated from conditionally healthy people.
Having strains with high adhesiveness may indicate that inflammation increases.

In the analysis of the obtained results it could be concluded that the selected strains are not highly active according to all criteria.

Of course, not-specific method cannot guarantee the final determination of the role of the selected strain as the causative agent of purulent - inflammatory processes, including peritonitis. Only the set of objective indicators, which include sensitivity to antibiotics and can determine the proportion of a particular strain of infection.

However pathogenicity factors would examine the cultural, morphological, and biochemical properties tinctorial 94 strains of $E$. coli extracted from children and adult patients with peritonitis and museum strains. The results that were obtained in the study of biological properties that made it possible to assign them to form E. coli.

The first step in the studying of the nature of the growth of E. coli on Endo medium. Colonies were evaluated with the following characteristics (size, shape, lifting above the surface, features of the edges, density, texture). Equally important is the color of the isolated colonies of E. coli on Endo medium that depends on differentiating components of the environment.

Because of $(18-24)$ hours of incubation, the medium Endo, E. coli colonies had the correct round shape, smooth, 
convex surface, smooth edge. The red colonies with a metallic shine on the nutritious medium were lactose positive colony. Pink or colorless colonies with a reddish center lackluster - strains which are poorly fermentated by lactose. Remained colorless colonies were not lactose fermentated.

To confirm the bacteria belonging to the family of products determined Eterobacteriaceae enzymes catalase and oxidase, subplanted colony on the combined environment for primary identification (Kligler Iron Agar).

The feedback list of the growth on the agar Kligler Iron Agar was carried through (18-24) h incubation, herewith noted the presence of gas generation, hydrogen sulphide production, fermentation of lactose and glucose. Fermentation of glucose and lactose was determined to change the colour of the environment in the column and the beveled part. After the second phase of identifying those selected crops that fermented acids and glucose to only gas or acid fermented or not fermented lactose and no hydrogen sulfide produced.

To determine the biochemical properties isolated strains took a minimum number of differentiating tests (Table II).

One of the "key" biochemical tests for the identification of bacteria of the genus Escherichia is the ability to dispose of sodium acetate. But there are «inactive» E. coli, which gives a negative result in the majority of use tests, making them similar to Shigella and hafnium. Therefore, it is appropriate to determine the biochemical properties in the fullest possible extend but not only to evaluate single key tests.

Depending on the presence of these or other pathogenicity factors, serological markers and epidemiological features, diarrhea $E$. coli are divided into the following categories: enterotoxigenic (ETEC), enteroinvasive (EIEC), enteropathogenic (EPEC), enterohaemorrhagic (EHEC) And enteroaggregate or enteroadergic E. coli (enteroaggregative, enteroadherent - EaggEC, EAEC) [2,3].

With a significant increase in the $E$. coli concentration in the intestinal biotype, the translocation of microorganisms into other organs and systems is possible, with such changes the coliform biotope can serve as an endogenous source of high - valued clones of conditionally pathogenic microorganisms capable of reducing the colonization resistance of individual biotopes ${ }^{1}$. Among the infections of the extracurricular localization that can cause the colon, distinguish the infection of the urinary and respiratory tract, intra-abdominal, skin and soft tissues, as well as generalized $[2,3]$.

In our study, the pathogenic species of the esherichia do not actually differ from the representatives of the normal microflora according to their morphological, biochemical and cultural properties. Therefore, it was necessary to carry out serological diagnostics for the identification of pathogenic serotypes. The presence in the pathological material of E. coli of a certain OK group was determined with a positive result of the agglutination reaction of live cultures with the corresponding monovalent OK-serum, as well as the proven cultures - with adsorbed O-serum.

In the study of serological properties of selected strains of $E$. coli $(\mathrm{n}=94), 65.8 \%$ of pathogenic serotypes were de- tected. At the same time, all isolated E. coli isolated from the abdominal cavity in children and adults, as well as museum strains belonged to the EPCP (O127: K63, O33: $\mathrm{K}-)$ and 1 to EICP (O144: K-).

\section{CONCLUSIONS}

In our investigation pathogenic pieces of Esherihies are virtually in distinguish able from representatives of normal microflora on its morphological, biochemical and cultural properties. During investigation of serological properties of selected of E.coli strains $(n=94) 65.8 \%$ of pathogenic serotypes were revealed. Moreover, all marked E.coli isolated from the abdominal cavity of children and adults, as well as museum strains related to enteropathogenic E. coli (O127: K63, O33: $\mathrm{K}-)$ and 1 -to enteroinvazive Escherichia coli (O144: K-).

\section{Abbreviations:}

ExPEC - Extraintestinal pathogenic Escherichia coli, E.coli - Escherichia coli,

A - acid, G - gas.

\section{REFERENCES}

1. Hooper LV., Gordon Jl. Commensal host-bacterial relationship in the gut. Science, 2001, 292, 1115-8.

2. Murray PR. Human microbiota. In: Borriello SP, Murray PR, Funke G, eds. Topley and Wilson`s Microbiology and Microbial Infections: Bacteriology. 10th edition. London: HodderArnold, 2007.

3. Donnenberg, M. S. 2002. Escherichia coli: virulence mechanisms of a versatile pathogen, Elsevier Science Academic Press, San Diego, Calif.

4. Kaper JB, Nataro JP, Mobley HL. Pathogenic Escherichia coli. Nat Rev Microbiol 2004; 2:123-40.

5. Russo TA, Johnson JR. Proposal for a new inclusive designation for extraintestinal pathogenic isolates of Escherichia coli: ExPEC. J InfectDis 2000;181:1753-1754.

6. Johnson, J.R., andT.A. Russo. 2002. Extraintestinal pathogenic Escherichia coli (ExPEC): the "other bad E. coli."J. Lab. Clin. Med. 139:155-162

7. Croxen MA, Law RJ, Scholz R, Keeney KM, Wlodarska M, Finlay BB. Recent advances in understanding enteric pathogenic Escherichia coli. Clin Microbiol Rev. 2013; 26(4):822-880.

8. Szeto CC, Chow VC, Chow KM, Lai RW, Chung KY, Leung CB, et al. Enterobacteriaceae peritonitis complicating peritoneal dialysis: a review of 210 consecutive cases. Kidney Int 2006; 69:1245-52.

9. Brilis V. I. Metodika izucheniya adgezivnogo proczessa mikroorganizmov [Method for studying the adhesive process of microorganisms]. Laboratornoe Delo. 1986; 4: 210-212 (in Russian).

10. Bücker R, Schulz E, Günzel D et al. a-Haemolysin of Escherichia coli in IBD: a potentiator of inflammatory activity in the colon. Gut 2014; 63 : 1893-1901.

11. Burgos Y, Beutin L. Common origin of plasmid encoded alpha-hemolysin genes in Escherichia coli. BMC Microbiol 2010; 10: 110-193.

12. Schmidt $\mathrm{H}$, Karch H. Molecular analysis of the plasmid-encoded hemolysin of Escherichia coli 0157:H7 strain EDL 933. Infect. Immun. 1995; 63 (3):1055 - 1061.

13. Martinez-Medina M, Aldeguer X, Lopez-Siles M et al. Molecular diversity of Escherichia coli in the human gut: new ecological evidence supporting the role of adherent-invasive E. coli (AIEC) in Crohn's disease. Inflamm Bowel Dis 2009; 15: 872-882. 


\section{ORCID and contributionship}

Olha Yu. Kosilova - 0000-0001-6558-0710 A,B,C,D,E,F

Oleksandra O. Vovk-0000-0002-0649-3163 C,D,E,F

Nataliia M. Katelevska - 0000-0001-5362-2979 E,F

Tetiana P. Osolodchenko - 0000-0001-7258-3880 E,F

Svitlana V. Ponomarenko - 0000-0003-3994-3500 E,F

Viacheslav Yu. Vdovichenko - 0000-0002-7374-9990 E,F

\section{Conflict of interest:}

The Authors declare no conflict of interest

\section{CORRESPONDING AUTHOR}

Oleksandra 0. Vovk

Kharkiv National Medical University,

4 Nauky Avenue, 61022, Kharkiv, Ukraine

e-mail:vovkalexandra80@ukr.net

Received: 28.04 .2019

Accepted: 14.11 .2019

A - Work concept and design, B - Data collection and analysis, C - Responsibility for statistical analysis,

D-Writing the article, $\mathbf{E}$-Critical review, $\mathbf{F}$ - Final approval of the article 